\title{
FLOW IN ROOF GUTTERS
}

\author{
By K. Hilding Beij
}

ABSTRACT

The design of roof gutters, heretofore, has been solely a matter of judgment based on experience. In order to provide a more rational method, an experimental study of the basic problem of roof gutters was undertaken. This problem may be stated as follows: What is the capacity of a straight open trough of uniform cross section, one end closed and the other end discharging freely, when the quantity of water flowing past a given cross section is directly proportional to the distance of the cross section from the closed end of the trough? Tests were made on rectangular and semicircular gutters of various lengths and widths, both level and sloping, and on one gutter of irregular cross section. Level gutters only are discussed in this paper.

Empirical formulas are given for determining capacity of level rectangular and semicircular gutters, and graphs for rapid solution are presented. An approximate method for gutters of irregular cross section, based on the tests of one such gutter, is also given.

Theoretical formulas for level gutters or channels of rectangular, triangular, trapezoidal, and semicircular cross sections are derived. 'The formulas for rectangular channels are compared with the results of the tests.

CONTENTS

I. Introduction

II. Apparatus

III. Test gutters

IV. Experimental procedure

1. Adjustment of gutter levels

2. Zero readings of piezometers

3. Water measurement

4. Observations

V. Experimental results

1. Data

2. Flow distribution

VI. Empirical formulas for capacity

1. Semicircular gutters._.

2. Rectangular gutters

3. Application of formulas

(a) Semicircular gutters

(b) Rectangular gutters

(c) Gutters of irregular cross section

(d) Factor of safety

(e) Sloping gutters

(f) Leader connections

VII. Theoretical analysis

1. General equation of flow

2. Level gutters without friction

3. Evaluation of $H$

(a) Rectangular

(b) Triangular

(c) Trapezoidal

(d) Semicircular

4. Equations for particular cases

(a) Rectangular.

(b) Triangular

(c) Trapezoidal

(d) Semicircular.

5. Comparison of theoretical results with experimental data--

VIII. Summary 


\section{INTRODUCTION}

The investigation which forms the subject of this paper was one of the problems included in a research on copper roofing conducted jointly by the Copper and Brass Research Association and the Bureau of Standards.

The purpose of the investigation was to provide a more satisfactory basis for the design of roof gutters and leader connections than the arbitrary and widely variant rules now used. Because of limitations of time and facilities, the investigation was confined to the basic problem of roof gutters, which may be stated as follows: What is the capacity of a straight, open trough of uniform cross section, one end closed and the other end discharging freely, when the quantity of water flowing past a given cross section is directly proportional to the distance of the cross section from the closed end of the trough?

Because of the complex nature of the flow in sloping roof gutters, satisfactory formulas have not been developed from the experimental results. Accordingly, this paper deals only with level gutters.

\section{APPARATUS}

Figure 1 shows the manner in which gutters were mounted for test, the method of supplying water to the gutters, and the type of piezometer used. The pipe layouts for large and small flows are shown in

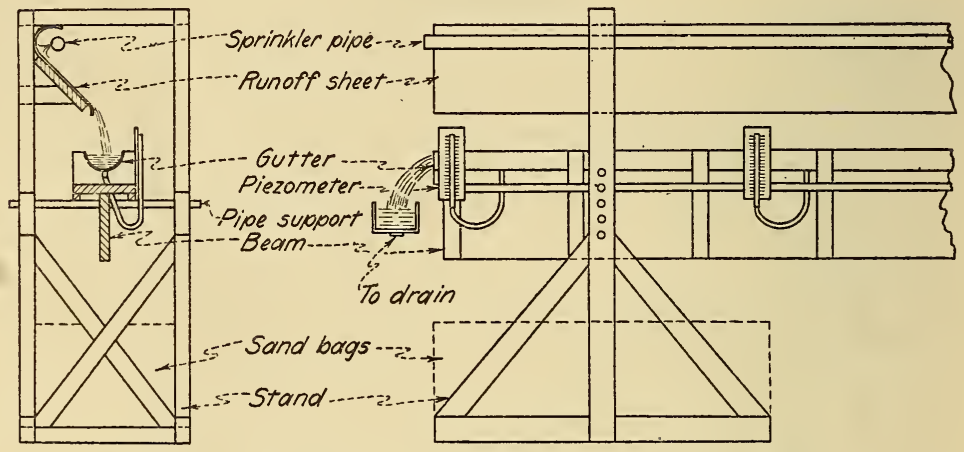

FigURE 1.-Sketch of apparatus for tests of roof gutters.

figure 2. A 2-inch Hersey water meter, equipped with a 10 cubic-foot dial divided at 1 cubic-foot intervals and rated at the Bureau of Standards, was used.

\section{TEST GUTTERS}

A descriptive list of the level gutters which were tested is given in table 1. Note that some of the 6 -inch gutters were tested with outlets instead of freely discharging from the end. In figure 3 are given drawings of the outlets used and sketches showing the position of outlets under the various test conditions. The form of cross section of the molded gutter, no. 27 , in table 1 , is shown in figure 4 . 


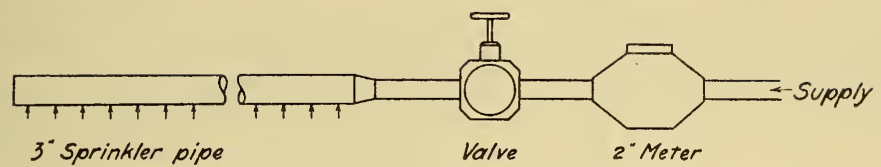

A. Arrangement for Tests on 6-inch Gutters

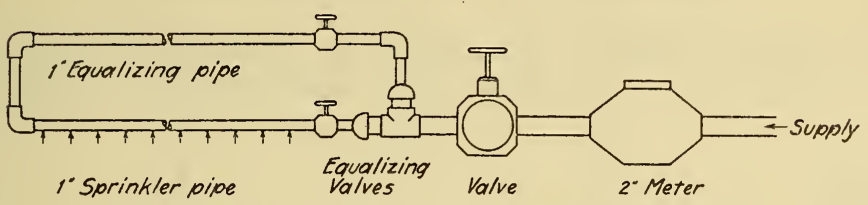

B. Arrangement for Tests on 3-inch and 4-inch Gutters

Figure 2.-Diagram of piping for tests of roof gutters.

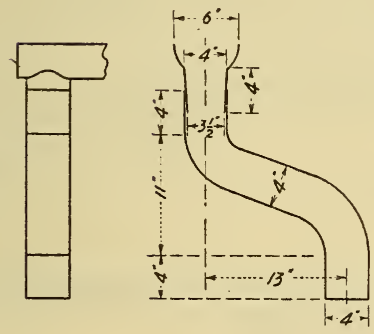

A. Outlet and Leader Connection for 6-inch Semicircular Gutter
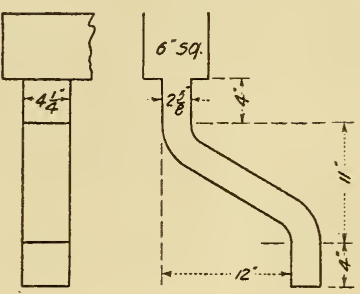

8. Outlet and Leader Connection for Rectangular and Molded Gutters

\section{$\square=-2=$ \\ Discharging freely at one end}

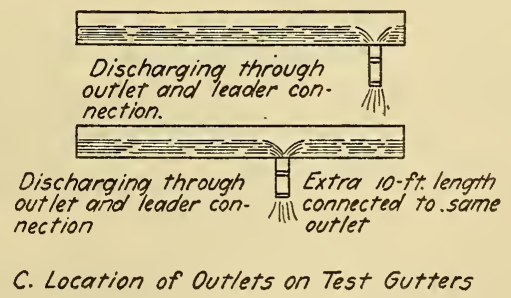

FIGURE 3.-Outlets and leader connections used in tests of roof gutters. 


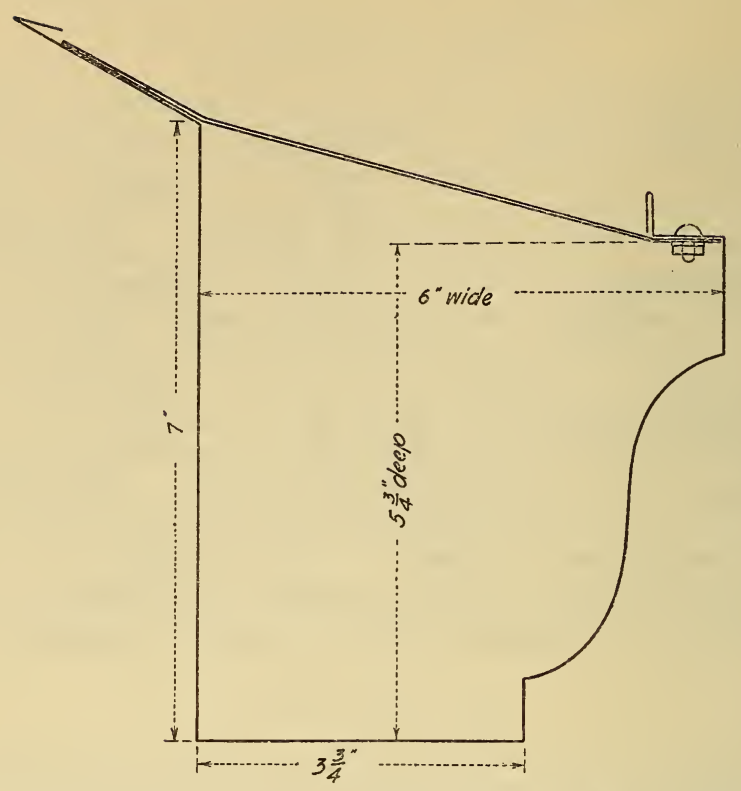

FIGURE 4.-Cross section of molded gutter (no. 27 in table 1).

Table 1.-Descriptive list of level gutters tested.

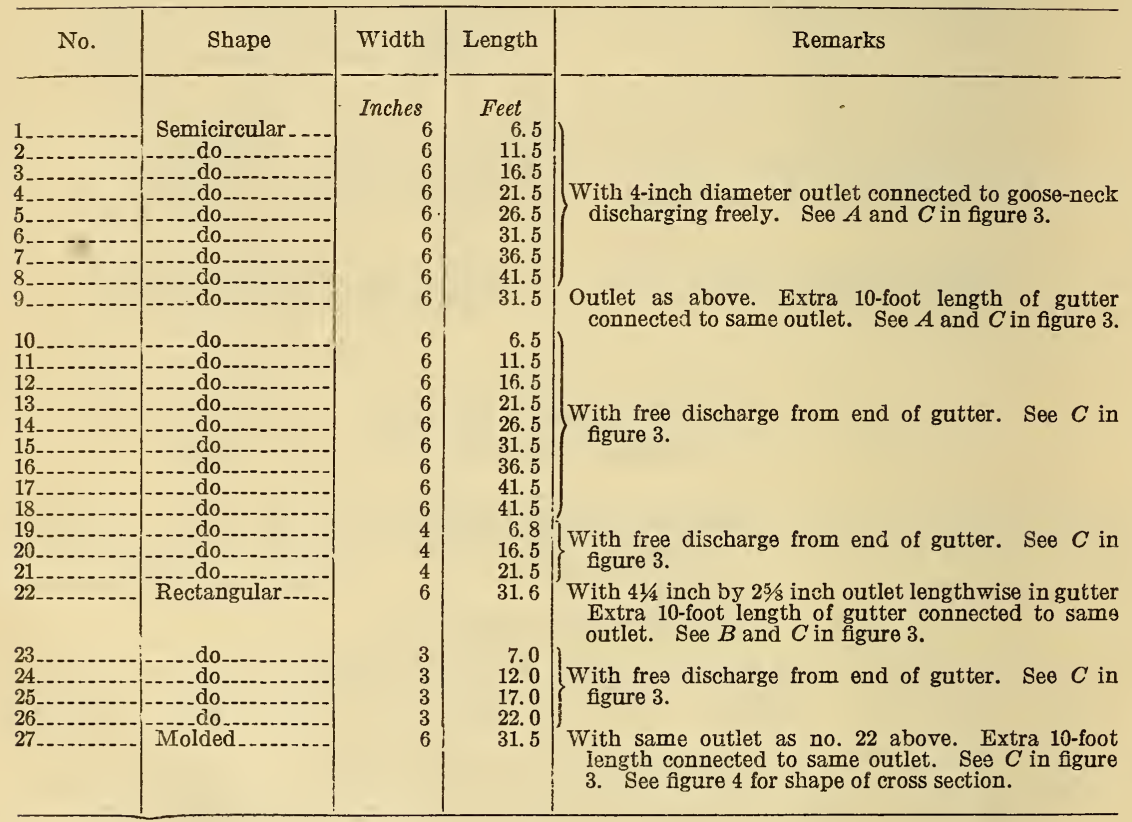




\section{EXPERIMENTAL PROCEDURE}

\section{ADJUSTMENT OF GUTTER LEVELS}

Final adjustments of the gutter levels were made with reference to a water surface. The gutter was partially filled with water and depths were measured at intervals of 30 inches along the center line with a point gage. The gutter was adjusted until the depths were as nearly uniform as possible.

\section{ZERO READINGS OF PIEZOMETERS}

The differences between the piezometer readings and the average depth of water measured with a point gage as before gave the zero readings for the piezometers.

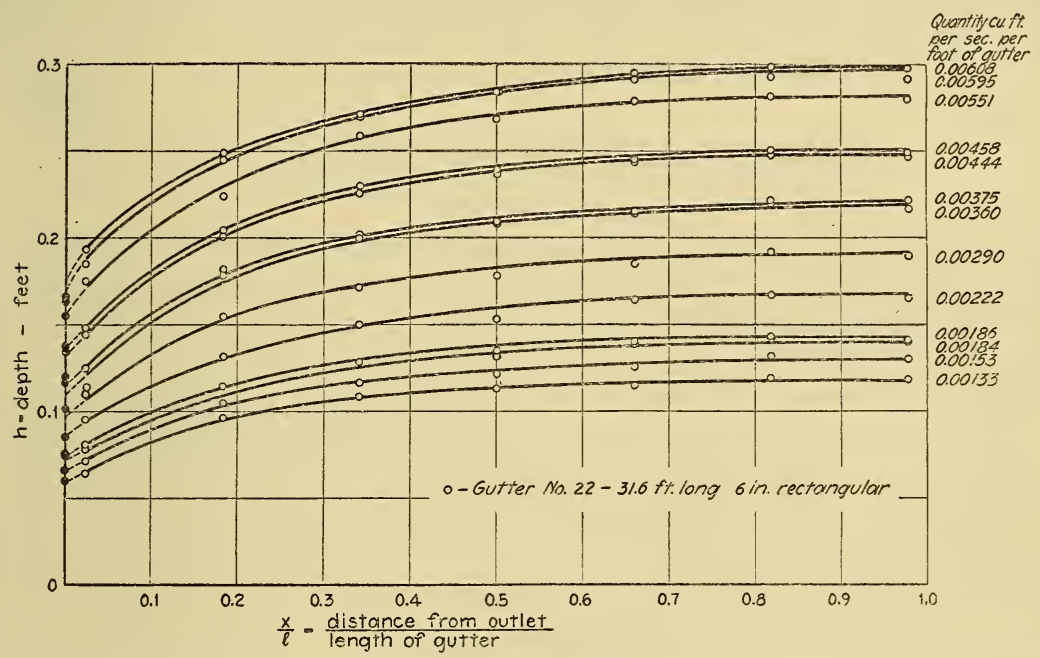

FIGURE 5.-Depths of water in 6-inch rectangular gutter for various rates of flow. The solid black points represent computed values for the depth at the point of outflow.

\section{WATER MEASUREMENT}

In general, the time required for a flow of 10 cubic feet as indicated by the meter was measured by a stop watch. For very low rates of flow the time for 5 cubic feet was measured. The rate of flow thus determined, corrected according to the meter calibration curve and divided by the length of the perforated pipe, gave the inflow per foot of gutter. The error in the corrected meter reading did not exceed one half of 1 percent.

\section{OBSERVATIONS}

A typical test run consisted of 15 observations on each piezometer as follows: The flow was adjusted to the lowest rate practicable and a few minutes allowed for it to become steady. Then all piezometers were read while the rate of flow was being measured. This set of readings was repeated, and the averages of the two sets constituted one observation. 


\section{EXPERIMENTAL RESULTS}

\section{DATA}

The data are too voluminous for complete presentation. Therefore, only the data for one 6-inch rectangular and part of the data for two 6-inch semicircular gutters are given in figures 5 and 6 .

\section{FLOW DISTRIBUTION}

The curves in figure 6 give an indication of the uniformity of flow from the sprinkler pipe. In obtaining these results a single long gutter was used, separated into two independent sections of equal length by a partition at the middle. ${ }^{1}$ As shown by the graph, the results on the two gutters differed slightly. However, the differences were small in most cases and did not exceed about 5 percent in the

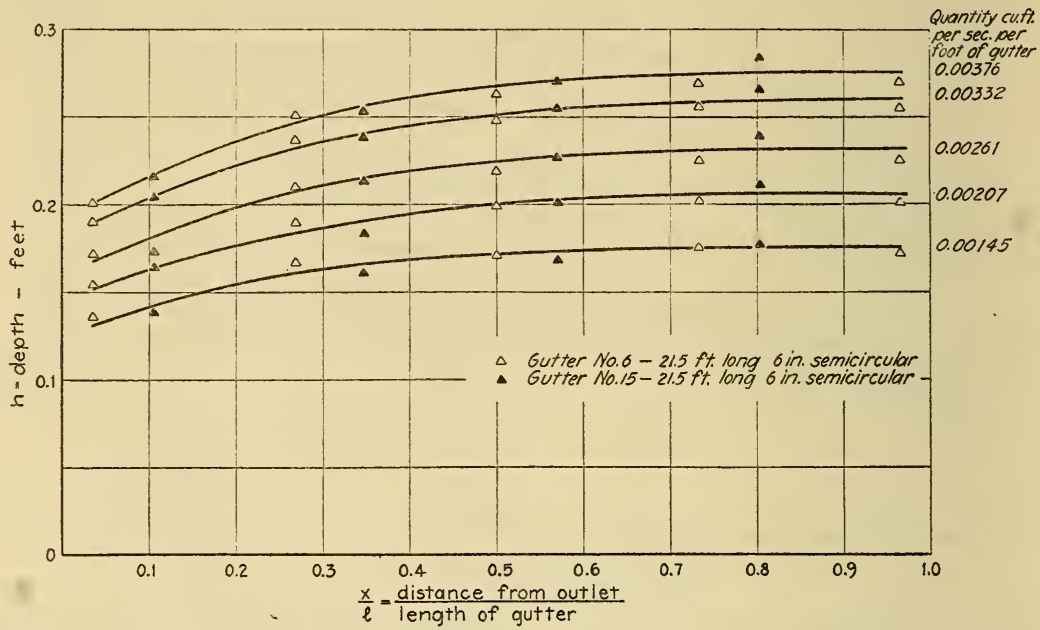

FIGURE 6.-Depths of water in 6-inch semicircular gutters for various rates of flow.

worst case. For simplicity, therefore, it was assumed that the discharge was uniformly distributed. This assumption implies that the average curves shown in figure 6 represent the results on both gutters.

\section{EMPIRICAL FORMULAS FOR CAPACITY}

\section{SEMICIRCULAR GUTTERS}

The following notation will be used, and all quantities expressed in units of feet and seconds.

$$
\begin{aligned}
& l=\text { length of gutter. } \\
& b=\text { width of gutter. } \\
& x=\text { distance from outlet to any point on gutter. } \\
& h=\text { depth of water. } \\
& q=\text { inflow per foot of gutter per second. } \\
& Q=\text { total inflow }(Q=q l) \text {. } \\
& g=\text { acceleration of gravity. }
\end{aligned}
$$

1 This method of securing tests on two gutters simultaneously was adopted for nearly all of the experiments on level gutters. 
The problem is to determine from the experimental data the depth of water $h$ at any desired distance $x$ from the outlet of a gutter in terms of the dimensions of the gutter, the physical properties of water, and the amount of inflow; and in particular, the depth at the upper end of the gutter, since this determined the capacity.

The physical properties of water which may be involved in the problem are

and

$$
\begin{aligned}
\mu & =\text { viscosity } \\
\rho & =\text { density } \\
\tau & =\text { surface tension. }
\end{aligned}
$$

To take account of the roughness of the gutter surface, we can introduce a friction coefficient such as the dimensionless factor $f$.

Then $h=$ function $(l, b, x, q, g, \mu, \rho, \tau, f)$,

where the form of the function is to be determined so as to be in agreement with the experimental data. By dimensional analysis, ${ }^{2}$ the number of variables may be reduced and equation (1) becomes

$$
\left(\frac{h}{l}\right)=F\left[\left(\frac{q^{2}}{l^{3} g}\right),\left(\frac{q \rho}{\mu}\right),\left(\frac{l \tau}{\rho q^{2}}\right),(f),\left(\frac{b}{l}\right),\left(\frac{x}{l}\right)\right],
$$

where $F$ is a function (as yet undetermined) of the six dimensionless variables in the bracket. The first of these variables corresponds to the Froude number and the second to the Reynolds number. The third has no generally used name but may be called the "surface tension number." The fourth is the roughness coefficient and the others are shape factors.

We will assume for simplification that the Reynolds number, the surface tension number and the friction coefficient may be neglected in deriving the general equation for the gutters. This implies that the Reynolds number is so high that the energy losses are almost wholly determined by the turbulance of the flow. This assumption is arbitrary and can be justified only by the results obtained. Also, even if we find the assumption to be legitimate within the range of the experiments, it must be borne in mind that if the range is extended (to larger or smaller gutters, to different liquids, or to gutters with different roughness) the results may not be applicable. With this assumption equation (2) simplifies to

$$
\left(\frac{h}{l}\right)=F\left[\left(\frac{q^{2}}{l^{3} g}\right),\left(\frac{b}{l}\right),\left(\frac{x}{l}\right)\right] .
$$

From the data it was found that equation (3) could be replaced with a close approximation by

$$
\left(\frac{h}{l}\right)=\left(\frac{q^{2}}{l^{3} g}\right)^{\frac{1}{3}}\left(\frac{b}{l}\right)^{-\frac{1}{1}} F_{1}\left(\frac{x}{l}\right),
$$

where $F_{1}$ represents an undetermined function. Equation (4) may be rewritten

$$
h\left(\frac{g b}{q^{2} l^{2}}\right)^{\frac{1}{2}}=F_{1}\left(\frac{x}{l}\right) .
$$

${ }^{2}$ E. Buckingham, Model experiments and the forms of empirical equations. Trans. A.S.C.E., vol. 37 , 1915. 
Values of the left-hand member of equation (5) computed from the data are plotted in figure 7 as a function of $\left(\frac{x}{l}\right)$. Each point represents the result of the 15 observations at one piezometer on one gutter.

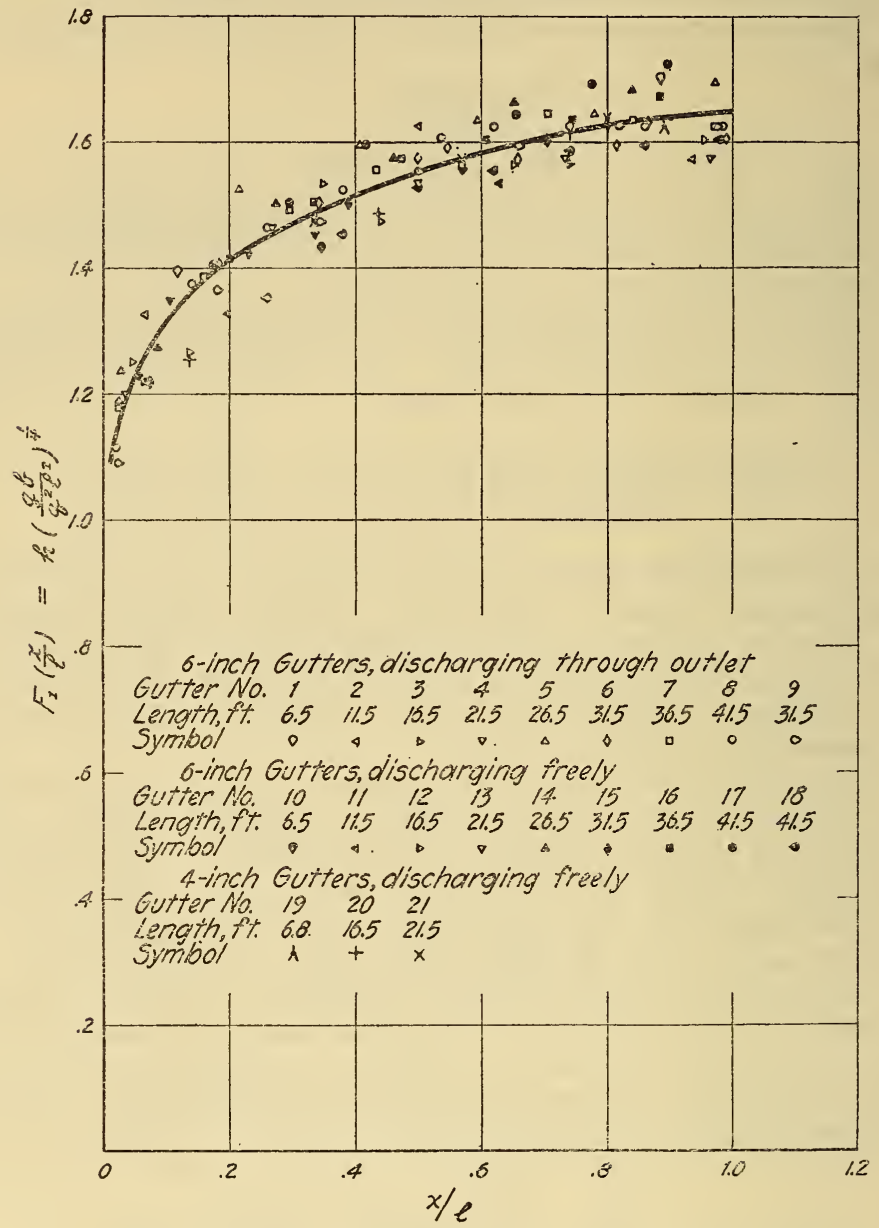

FIGURE 7.-Values of $F_{1}\left(\frac{x}{l}\right)=h\left(\frac{g b}{q^{2} l^{2}}\right)^{\frac{1}{3}}$ for semicircular gutters. The approximation curve is given by the equation $F_{1}\left(\frac{x}{l}\right)=1.65\left(\frac{x}{l}\right)^{\frac{2}{10}}$.

The curve drawn in figure 7 , which is a graph of the equation,

$$
F_{1}=1.65\left(\frac{x}{l}\right)^{\frac{1}{10}} \text {, }
$$

is adopted as an approximate representation of the plotted points. Substituting this value of $F_{1}$ in equation (5) and solving for $h$, there results

$$
h=1.65\left(\frac{q^{2} l^{2}}{g b}\right)^{\frac{1}{4}}\left(\frac{x}{l}\right)^{2^{2} \frac{1}{6}},
$$


which is the complete formula for flow in level semicircular roof gutters.

To obtain a formula for capacity, it should be noted that the maximum depth occurs at the upper or closed end. If the depth of water here is equated to the total depth of the gutter, then substituting in (8) the following values;

$$
h=\frac{b}{2}, x=l, Q=q l, g=32.2,
$$

there is obtained finally

$$
Q=0.52 b^{\frac{s}{2}},
$$

which gives the capacity in cubic feet per second of a semicircular level gutter in terms of its width in feet. Inverting equation (8), there results

$$
b=1.3 Q^{\frac{2}{5}}
$$

which gives the width in feet of a semicircular level gutter required for a known flow.

\section{RECTANGULAR GUTTERS}

Similarly, there.was found for rectangular gutters the equation

$$
\left(\frac{h}{l}\right)=\left(\frac{q_{i}^{2}}{l^{3} g}\right)^{\frac{x}{16}}\left(\frac{b}{l}\right)^{-\frac{3}{l}} F_{1}\left(\frac{x}{l}\right)
$$

which may be written

$$
h\left(\frac{g^{5} b^{12}}{q^{10} l^{13}}\right)^{\frac{1}{16}}=F_{1}\left(\frac{x}{l}\right) .
$$

Values of the left-hand member of equation (10) computed from the data are plotted in figure 8 . The curve, which is a graph of the equation

$$
F_{1}=0.823\left(\frac{x}{l}\right)^{\frac{2}{8}}
$$

is adopted as an approximate representation of the plotted points.

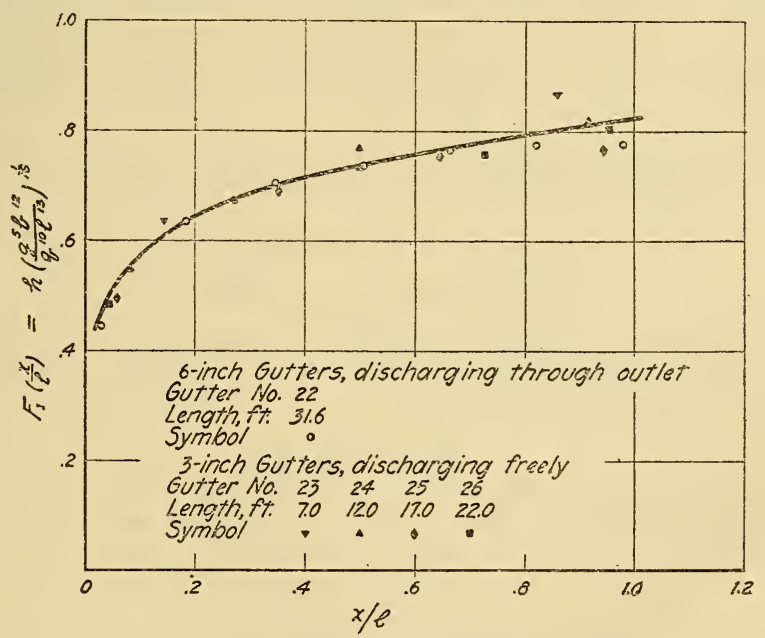

FIGURE 8.-Values of $F_{1}\left(\frac{x}{l}\right)=h\left(\frac{g^{5} b^{12}}{q^{107^{13}}}\right)^{\frac{1}{16}}$ for rectangular gutters. The approximation curve is given by the equation $F_{1}\left(\frac{x}{l}\right)=0.823\left(\frac{x}{l}\right)^{\frac{l}{l}}$. 
Substituting this value of $F_{1}$ in equation (11) and solving for $h$, there results

$$
h=0.823\left(\frac{q^{10} l^{13}}{g^{5} b^{12}}\right)^{\frac{1}{16}}\left(\frac{x}{\bar{l}}\right)^{\frac{1}{6}},
$$

which is the complete formula for flow in level rectangular roof gutters.

It is convenient to deal with the ratio of the depth of a rectangular gutter to its width, as follows:

$$
m=\frac{\text { depth of gutter }}{\text { width of gutter }}
$$

Then for a gutter carrying its maximum flow, we have as before:

$$
h=m b, x=l, Q=q l, g=32.2,
$$

so that equation (12) reduces to

and

$$
Q=7.75 m^{\frac{8}{3}}\left(\frac{b}{l}\right)^{\frac{3}{t \sigma}} b^{\frac{5}{2}}
$$

$$
b=0.483 m^{-\frac{4}{8} l^{38}} Q^{\frac{5}{4}} .
$$

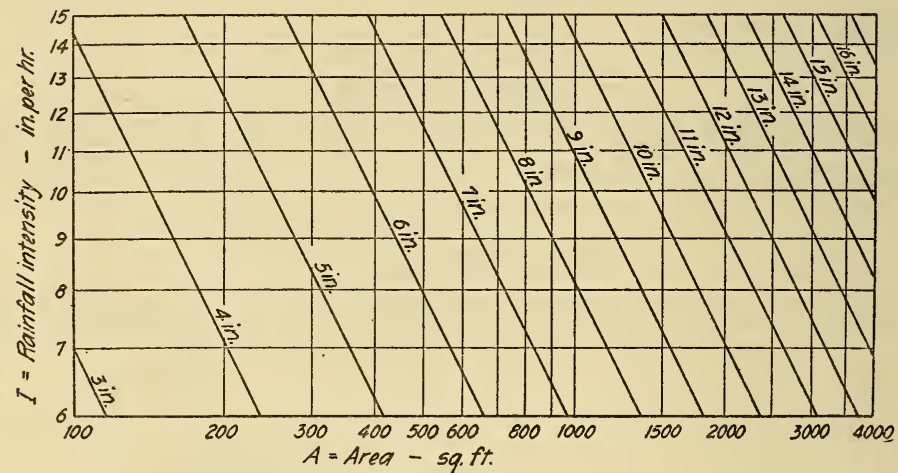

FIGURE 9.-Graph for determining the size of a level semicircular gutter when the roof area and the rainfall intensity are known.

\section{APPLICATION OF FORMULAS}

(a) SEMICIRCULAR GUTTERS

If the maximum rainfall intensity in inches per hour be denoted by $I$, then the total quantity in cubic feet per second falling on a roof of area A square feet will be

$$
Q=2.31 \times 10^{-5} I A \text {. }
$$

Substituting this value of $Q$ in equation (8a) for semicircular gutters, the result is

$$
b=0.0182(I A)^{\frac{2}{5}},
$$

which gives the width in feet of gutter required when the rainfall intensity and the roof area are known. The formula is conveniently solved by means of a graph such as that in figure 9 . 
In the graph, the gutter widths are expressed in inches as this is the customary unit. By roof area, the area of the horizontal projection of the roof is understood; that is, roof areas should be computed from the roof plans. If the roof is not rectangular, the dimensions of the rectangle enclosing the plan may be taken instead of the actual roof dimensions, provided the inflow is nearly uniformly distributed along the gutter.

\section{(b) RECTANGULAR GUTTERS}

By substitution of equation (14) in equation (13a) there is obtained for rectangular gutters the formula

$$
b=0.0106 m^{-\frac{4}{3}} l^{\frac{3}{2}}(I A)^{\frac{5}{14}},
$$

which can be readily solved with the aid of the graphs in figures 10 and 11.

\section{(c) GUTTERS OF IRREGULAR CROSS SECTION}

The required sizes of level gutters of other than semicircular or rectangular shapes can be determined approximately by finding the semicircle or rectangle of the same area which most closely fits the irregular cross section as in figure 12 .

This method of approximation for gutters of irregular cross section was checked by data obtained on a molded copper gutter. For each depth of flow, the width of a rectangle of the same area as the filled part of the cross section was determined. The extreme cases are shown by (c) and (d) in figure 12 . From these dimensions and the length of the gutter, the corresponding computed values of the quantity $(I A)=Q$ were determined by means of the graphs in figures 10 and 11 . These computed values were compared with observed values determined from the known quantity of flow in each case. The re-

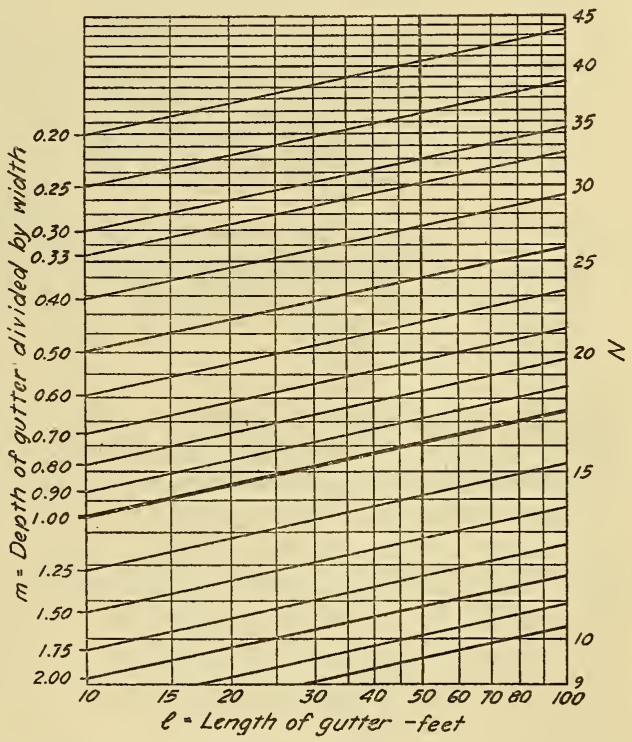

FIGURE 10.-Graph for determining the size of a level rectangular gutter when the roof area and the rainfall intensity are known. To be used in conjunction with the graph in figure 11. sults are plotted on the graph in figure 13. If the values found by both methods agreed exactly, the points represented by the circles would fall on the straight line. It is evident that the discrepancies are very small except for three points, which may very likely be affected by experimental error.

\section{(d) FACTOR OF SAFETY}

The annoyance and damage resulting from occasional overflows of over-loaded gutters depend upon the character of the building under consideration, and their importance is a matter of judgment which 
must be left to the architect or designing engineer. The duration and intensity of heavy rains and the frequency with which they may be expected to occur may be ascertained for any given locality by

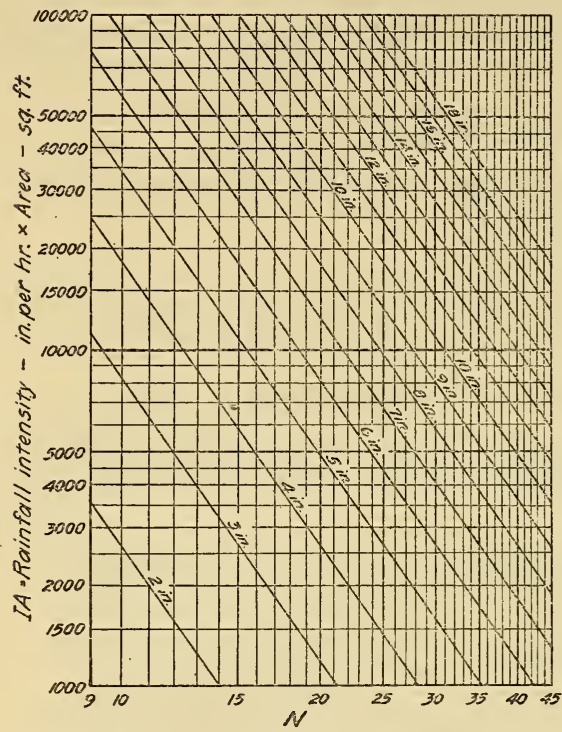

FIGURE 11.-Graph for determining the size of a level rectangular gutter when the roof area and the rainfall intensity are known. To be used in conjunction with the graph in figure 10. application to the nearest station of the United States Weather Bureau or the local forecaster. Then the intensity to be used in design may be chosen according to circumstances. The intensity should be picked in each case high enough so that the gutter dimensions determined from the graphs can be used directly without multiplying by some arbitrary "factor of safety."

\section{(e) SLOPING GUTTERS}

Sloping gutters carry much more water than level gutters of the same size. The graphs for level gutters may be used, however, to check dimensions chosen for sloping gutters. If the selected dimensions are greater than those given by the charts, it is obvious that the gutter will be unnecessarily large.

\section{(f) LEADER CONNECTIONS}

The usual cause of overflowing gutters is insufficient capacity of leader connections. Gutter outlets are very commonly too small, and may be further obstructed by strainers and also leaves or other debris. It is suggested that outlets should be as nearly the full width of the gutter as possible and should extend along the gutter for a length equal to the total depth of the gutter. From the outlet a tapering connection, of length not less than its maximum width, should lead to the conductor. If two gutters empty into the same leader, then the outlet should be half again as long as for a single gutter. For structural or architectural reasons, such outlets may be too large, in which case more leaders should be provided

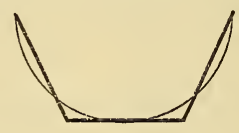

a

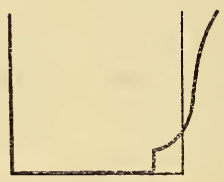

c

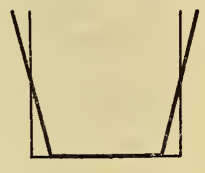

b

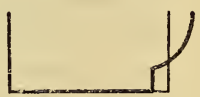

d

FIGURE 12.-Approximation of gutters of trapezoidal or irregular cross section by semicircles and rectangles.

and the gutter dimensions recomputed. These suggestions are based on the limited observations furnished by the tests, and therefore are subject to revision in the light of more complete information. 
When two outlets are provided at the ends of a single gutter, one half the gutter length and one half the roof area should be used in computing the gutter dimensions.

\section{THEORETICAL ANALYSIS}

\section{GENERAL EQUATION OF FLOW}

The empirical formulas which have been derived are adequate for determining the dimensions of roof gutters, because in this particular case only approximate results are needed. They should not, however, be applied to channels of dimensions widely different from those of the gutters tested, for in addition to the limitation introduced by the assumption of a high Reynolds number they are also only arbitrary functions of the remaining variables which have been worked out to fit the experimental results. Beyond the limits of the experiments these functions may still be valid, but it is also possible that they may diverge more and more from fact even within the limits of high Reynolds numbers and finally lead to results grossly in error. Furthermore, the formulas in themselves do not lead to any general results of interest. For these reasons and because the procedure used in deriving the formulas was inadequate for sloping gutters, a more detailed study was started. This study is not yet completed, but the results so far obtained are given here.

For convenience, the origin of

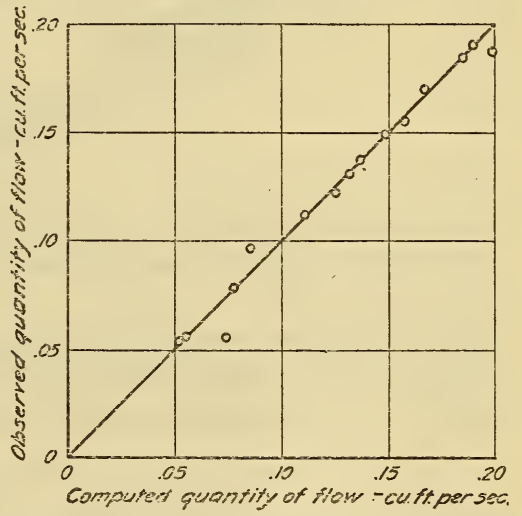

FIGURE 13.-Comparison of observed quantilies of flow with flows computed by approximation method for gutters of irregular cross sections. coordinates is taken at the upper end of the gutter instead of the outlet. Otherwise the same notation will be used, with the following additions:

$M=$ mass per unit time passing any cross section.

$q=$ volume inflow per unit time per unit length of gutter.

$w=$ specific weight of water.

$v=$ average velocity at any cross section.

$a=$ area of water cross section at any point.

$i=$ slope of gutter bottom.

$J=$ slope of water surface.

$f=$ frictional force per unit area of boundary of stream.

The following assumptions will be made:

1. As assumed in deriving the empirical equations, the effects of surface tension and viscosity may be neglected. This implies that the Reynolds number is so high that the energy losses are almost wholly determined by the turbulence of the flow. 
2. The mean velocity of the mass $M$ (or quantity $q x$ ) at any cross section is equal to the average velocity in the cross section ${ }^{3}$ and is given by the volume of flow divided by the area of the cross section $v=q x / a$.

3. Vertical components of velocity are negligible; since the slope $J$ of the water surface and the slope $i$ of the gutter are both small.

4. The water flowing into the gutter enters with a zero component of momentum in the direction of flow. This is equivalent to saying that the kinetic energy of the inflowing water is dissipated (as heat in eddies, etc.) and has no effect on the motion in the gutter.
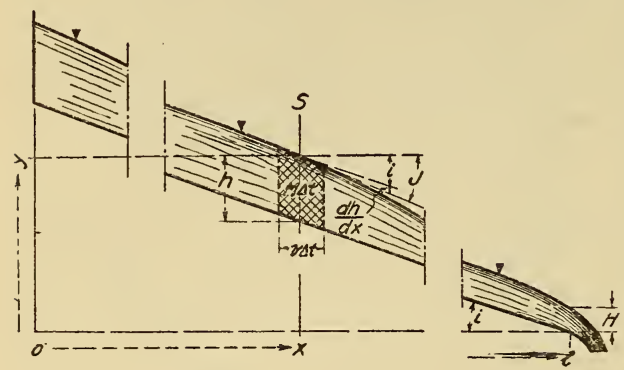

FIgURE 14.-Diagram of surface curve in roof gutter showing notation used in deriving theoretical formulas. forces, respectively, acting on the mass $M \cdot \Delta t$. Also the time rate of change of momentum is equal to the product of the space rate of change of momentum and the veloctiy, so that equation (17) becomes

$$
\frac{d}{d t}(M \cdot \Delta t \cdot v)=v \frac{d}{d x}(M \cdot \Delta t \cdot v)=G-F .
$$

The free surface of the mass $M \cdot \Delta t$. which is displaced a horizontal distance $\Delta x$, through the section $x$, drops by an amount $\Delta y$ so that its potential energy decreases by $M \Delta t \cdot g \cdot \Delta y$. This decrease, divided by $\Delta x$, gives the $x$-component of the effective force $G$ due to gravity, so that

$$
G=M \Delta t \cdot g \frac{d y}{d x}=M \Delta t \cdot g J
$$

Also,

so that, finally,

$$
J=i-\frac{d h}{d x}
$$

$$
G=M \cdot \Delta t \cdot g\left(i-\frac{d h}{d x}\right)
$$

\footnotetext{
${ }^{3}$ This is strictly true only when the relocity is constant over the entire cross section. Assuming uni width of stream, we have mean relocity $=\bar{v}=\int_{0}^{h} v^{2} d y / \int_{0}^{h} v d y$, where $v$ is some function of $y$ (the height above the stream bottom); and

$$
\text { average velocity }=v_{a}=-\frac{1}{h} \int_{0}^{h} v d y \text {. }
$$

It is evident that $\bar{v}$ is not equal to $v_{a}$ unless $v$ is a constant. Also $\bar{v}$ cannot be calculated unless the velocity distribution over the cross section is known.
} 
The frictional force, $F$, is assumed to depend on the velocity and the area of contact between the water and the sides and bottom of the gutter, as follows:

$$
F=f \frac{v^{2}}{2 g} p v \cdot \Delta t
$$

where $f \frac{v^{2}}{2 g}$ is the frictional force per unit area of contact; and $p$ is the wetted perimeter at the section $S$, so that $p v \cdot \Delta t$ is the area of contact.

By the substitution of (19) and (20) in (18), we have

Substituting

$$
v \frac{d}{d x}(M v)=M g\left(i-\frac{d h}{d x}\right)-f \frac{v^{2}}{2 g} p v
$$

we obtain finally

$$
M=\frac{q w x}{g} \text { and } v=\frac{q x}{a},
$$

$$
\frac{q^{2}}{g} \frac{d}{d x}\left(\frac{x^{2}}{a}\right)=a\left(i-\frac{d h}{d x}\right)-\frac{f q^{2}}{2 g w} \cdot \frac{p x^{2}}{a^{2}} .
$$

Equation (22) is the general equation for gutters or channels in which the quantity flowing at any section is proportional to the distance from the upper end. Note that both $a$ and $p$ are functions of the depth and such constants as are required to define the shape of the gutter. The equation is it stands, therefore, cannot be integrated. Solutions have been obtained, however, for certain special cases which are given below.

\section{LEVEL GUTTERS WITHOUT FRICTION}

If we assume that the frictional forces are sufficiently small so that their effects can be neglected and also that the gutters are horizontal, then equation (22) reduces to a much simpler form as follows. ${ }^{4}$

$$
\frac{q^{2}}{g} \frac{d}{d x}\left(\frac{x^{2}}{a}\right)=-a \frac{d h}{d x}
$$

Evidently, both members of the equation are perfect differentials ( $a$ being a function of $h$ only) and the solution is, accordingly,

$$
\frac{q^{2} x^{2}}{g a}=-\int_{0}^{x} a \frac{d h}{d x} d x=-\int_{h_{\mathrm{o}}}^{h} a d h,
$$

where $h_{\mathrm{o}}$ is the depth at $x=0$ (closed end of trough)

Let $H$ be the depth at the outlet end of the trough. (This depth is known from other considerations, as will be shown later.)

When $x=l, h=H$, and we can write

$$
\frac{q^{2} l^{2}}{g A}=-\int_{h_{0}}^{H} a d h
$$

4 This result expressed in a slightly different form has previously been obtained by Julian Hinds. See "Side Channel Spillways", Trans. A.S.C.E., vol. 89, p. 881, 1926. 
where $A$ is the cross-sectional area corresponding to $H$. Subtracting (25) from (24) and simplifying

$$
-\frac{g a}{q^{2} l^{2}} \int_{h}^{H} a d h-\frac{a}{A}+\frac{x^{2}}{l^{2}}=0,
$$

which is the general equation, friction being neglected, for level channels in which the quantity flowing at any point is proportional to the distance from one (closed) end.

\section{EVALUATION OF H}

So far, $H$ has been defined only as the depth at the point of outflow of the gutter. In order to make use of equation (26) it is necessary to find a method of evaluating this depth. If we assume that the flow always adjusts itself so as to make the total energy of the stream at the outflow section a minimum, then this section is a critical section and the depth $H$ can be computed. ${ }^{5}$ The experimental justification for this assumption is presented in part in figure 5 .

Critical depths, computed for the outlet section for each quantity of flow, are plotted as solid circles on the ordinate axis. The extensions of the surface curves by the dotted lines pass quite near the corresponding critical depths, indicating that the assumption is valid within the limits of experimental errors. Similar results were obtained for all of the gutters tested. A very close agreement cannot be expected, since the computed values are based on assumption that the curvature of the water surface is negligible, and this assumption is obviously not fulfilled in this particular case.

To evaluate the critical depth $H$, we note that the total energy of flow, $E$, at a cross section is the sum of the potential and kinetic energies, so that

$$
E=M g H+\frac{1}{2} M V^{2}
$$

computed with reference to the bottom or invert of the channel. The condition for a minimum is

or

$$
\frac{d E}{d H}=M g+M V \frac{d V}{d H}=0,
$$

At the point of outflow,

$$
g+V \frac{d V}{d H}=0
$$

$$
V=\frac{q l}{A}
$$

and substitution of this value in (27) gives

whence,

$$
g+\frac{q l}{A} \cdot \frac{d}{d H}\left(\frac{q l}{A}\right)=0
$$

$$
\frac{q^{2} l^{2}}{g}=\frac{A^{3}}{\left(\frac{d A}{d H}\right)} .
$$

B Handbook of Hydraulics, by H. W. King, McGraw-Hill Book Co., Inc., 1929. Hydraulics of Open Channels, by B. A. Bakhmeteff, McGraw-Hill Book Co., Inc., 1932. 
If we denote the width of the water surface by $B$,

so that

$$
\frac{d A}{d H}=B,
$$

$$
\frac{q^{2} l^{2}}{g}=\frac{A^{3}}{B}
$$

a general equation, applying to any form of cross-section.

In order to proceed further it is necessary to specify the shape of the gutter. A number of the more common shapes are treated below.

(a) RECTANGULAR

For a rectangular gutter of width $b$, we have

$$
\begin{gathered}
A=b H, \\
B=b,
\end{gathered}
$$

and hence from (28)

$$
\frac{q^{2} l^{2}}{g}=\frac{b^{3} \dot{H}^{3}}{b},
$$

or

$$
\mathrm{H}=\sqrt[3]{\frac{q^{2} l^{2}}{g b^{2}}},
$$

(b) TRIANGULAR

The gutter consists of two sides, with any slopes equal or unequal, meeting at any angle so that the vertex of the cross section is at the lowest point. Then the width of the water surface, $B$, is proportional to the depth, so that

$$
A=\frac{1}{2} k H^{2}
$$

and

$$
B=k H \text {, }
$$

where $k$ is a constant determined by the slopes of the sides. Hence from (28)

or

$$
\frac{q^{2} l^{2}}{g}=\frac{1}{8} \frac{k^{3} H^{6}}{k H}=\frac{1}{8} k^{2} H^{5},
$$

$$
H=\sqrt[5]{8 \frac{q^{2} l^{2}}{g k^{2}}}
$$

(c) TRAPEZOIDAL

The cross section is in the form of a trapezoid with one level side forming the bottom and two sides sloping upward, the slopes not necessarily equal.

Then

$$
\begin{gathered}
A=s H+\frac{1}{2} k H^{2}=H\left(s+\frac{1}{2} k H\right), \\
B=s+k H,
\end{gathered}
$$


where $s$ is the constant width of the bottom of the gutter, and $k$ is a constant depending upon the slopes of the sides. By substitution in $(28)$

$$
\frac{q^{2} l^{2}}{g}=H^{3} \frac{\left(s+\frac{1}{2} k H\right)^{3}}{s+k H},
$$

from which the value of $H$ may be computed for any specified case.

(d) SEMICIRCULAR

Let $r$ be the radius of the cross section. Then

$$
A=r^{2} \cos ^{-1}\left(\frac{r-H}{r}\right)-(r-H)\left(2 r H-H^{2}\right)^{\frac{1}{2}}
$$

Substituting in (28)

$$
B=2\left(2 r H-H^{2}\right)^{1 / 2}
$$

$$
\left.\frac{q^{2} l^{2}}{g}=\frac{\left[r^{2} \cos ^{-1}\left(\frac{r-H}{r}\right)-(r-H)\right.}{s\left(2 r H-H^{2}\right)^{\frac{1}{2}}}\left(2 r H-H^{2}\right)^{\frac{1}{2}}\right]^{3}
$$

\section{EQUATIONS FOR PARTICULAR CASES}

We now proceed to determine the form of equation (26) for the various particular cases.

(a) RECTANGULAR

From equation (26) by substitution of the results given by (29) to $(29 \mathrm{c})$, inclusive, we obtain

$$
\left(\frac{h}{H}\right)^{3}-3\left(\frac{h}{H}\right)+2\left(\frac{x}{l}\right)^{2}=0 .
$$

The maximum depth occurs at the upper end of the gutter where $x=0$, and is given by

$$
h_{0}=\sqrt{3} H .
$$

If the ratio of the depth to the width of the gutter is $m$, then the capacity $Q$ is

$$
Q=2.5 m^{\frac{3}{2}} b^{\frac{5}{2}}
$$

where $Q$ is in cubic feet per second and $b$ is in feet.

(b) TRIANGULAR

From (26) and (30) to (30c), inclusive, we have

$$
2\left(\frac{h}{H}\right)^{5}-5\left(\frac{h}{H}\right)^{2}+3\left(\frac{x}{l}\right)^{2}=0 .
$$

The maximum depth, at $x=0$, is given by

$$
h_{o}=\sqrt[3]{\frac{5}{2}} H
$$


and the capacity $Q$, by

$$
Q=\frac{0.935}{k^{3 / 2}} b^{5 / 2}
$$

where $Q$ is in cubic feet per second, $b$ is in feet, and $k$ is the ratio of the top width $b$ to the vertical depth of the channel.

(c) TRAPEZOIDAL

From equation (26) and equations (31) to (31b), inclusive, there results

$$
\begin{aligned}
& \frac{h(s+k H)\left(s+\frac{1}{2} k h\right)}{H^{3}\left(s+\frac{1}{2} k H\right)^{3}}\left[\frac{h^{2}}{2}\left(s+\frac{1}{3} k h\right)\right. \\
& \left.\quad-\frac{H^{2}}{2}\left(s+\frac{1}{3} k h\right)\right]-\frac{h\left(s+\frac{1}{2} k h\right)}{H\left(s+\frac{1}{2} k H\right)}+\left(\frac{x}{l}\right)^{2}=0,
\end{aligned}
$$

which reduces to equation (33) if we put $s=b$ and $k=0$, or to equation (34) if we put $s=0$.

The maximum depth $h_{0}$ occurs when $x=0$ and is given by the equation

$$
h_{o}^{2}\left(s+\frac{1}{3} k h_{o}\right)-\frac{H^{2}}{s+k H}\left(s+\frac{1}{3} k H\right)(s+k H)+2\left(s+\frac{1}{2} k H\right)^{2}=0,
$$

and the capacity by eliminating $H$ from equations (31b) and (35a) and solving for $Q$.

(d) SEMICIRCULAR

From equation (26) and equations (32) to (32b), inclusive, the equation for semicircular channels becomes

$$
\frac{a B}{A^{3}}\left[3\{A(r-H)-a(r-h)\}+2\left\{\left(\frac{b}{2}\right)^{3}-\left(\frac{B}{2}\right)^{3}\right\}\right]-3 \frac{a}{A}+3\left(\frac{x}{l}\right)^{2}=0,
$$

and for the maximum depth,

$$
\frac{B}{A^{2}}\left[3\left\{A(r-H)-a_{o}\left(r-h_{o}\right)\right\}+2\left\{\left(\frac{b_{o}}{2}\right)^{3}-\left(\frac{B}{2}\right)^{3}\right\}\right]-3=0 .
$$

If the channel is filled, $h_{0}=r, b_{0}=2 r$ and $a_{o}=\frac{\pi r^{2}}{2}$;

so that

$$
\frac{B}{A^{2}}\left[3 A(r-H)+2\left\{r^{3}-\left(\frac{B}{2}\right)^{3}\right\}\right]-3=0,
$$

and combining this result with (32b), the value of $Q$ may be computed. 


\section{COMPARISON OF THEORETICAL RESULTS WITH} EXPERIMENTAL DATA

In order to illustrate the degree of concordance between the theoretical formulas and the results of the experiments, the measured depths at the upper ends of the rectangular gutters are plotted in figure 15 against the corresponding depths computed by means of

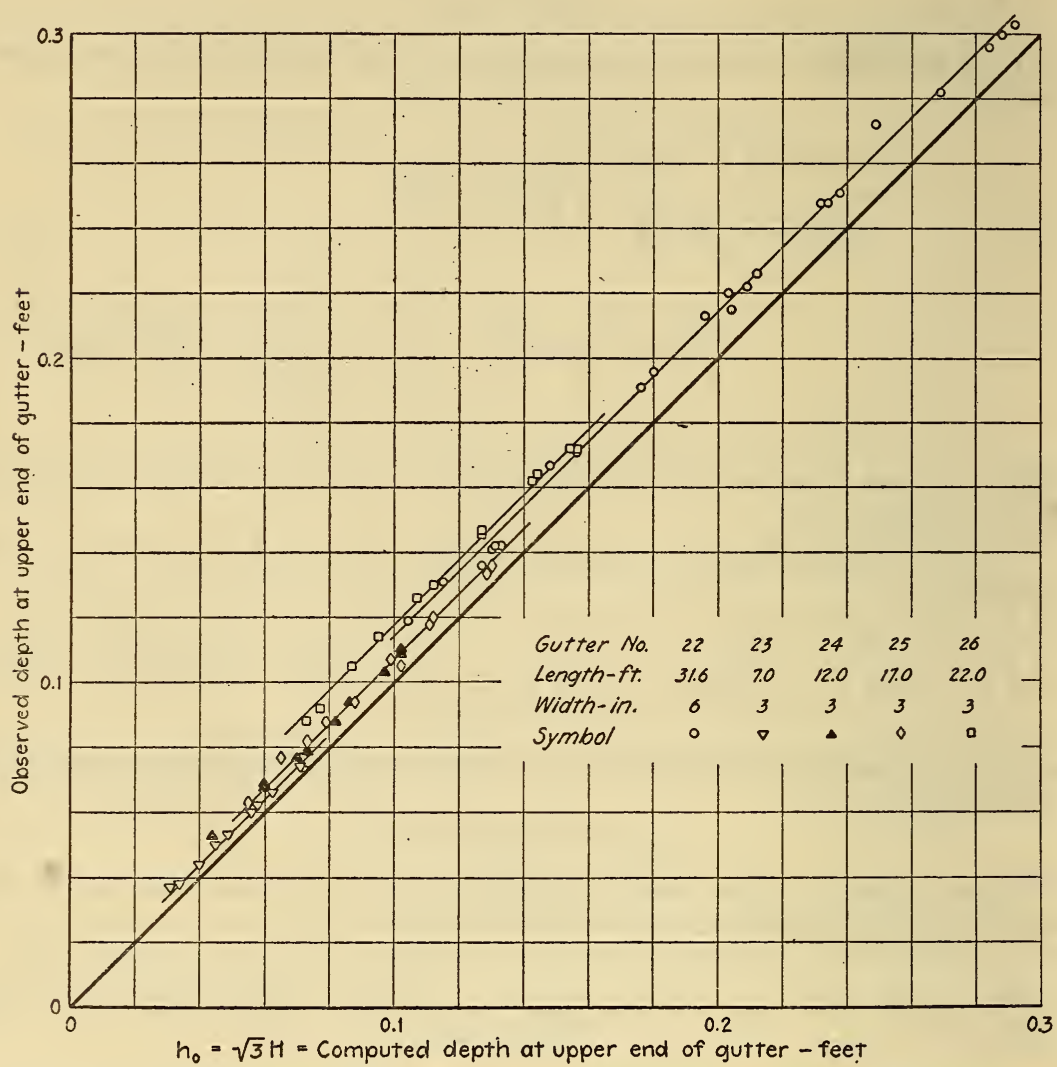

FIGURE 15.-Comparison of observed depths with depths computed from theoretical equation (33a) for 3-inch and 6-inch rectangular gutters.

equation (33a).

All of the observed depths are greater than the computed depths, since the resisting forces, such as friction, have been neglected in the derivation of the equation (33a).

For each gutter the difference between computed and observed depths is constant, or in other words, it is independent of the quantity flowing. The relative or percentage difference is, therefore, inversely proportional to the quantity flowing.

The results indicate that the differences between computed and actual depths for rectangular gutters depend on the gutter dimensions. The difference increases with increasing length and decreases with increasing width of gutter.

Further details will be left for future publication. 


\section{SUMMARY}

1. Empirical formulas for the capacity of level semicircular and rectangular roof gutters have been deduced from the results of tests. Graphs for simplifying the solutions of the formulas are presented.

2. An approximate method for determining the capacity of level roof gutters of irregular cross section is suggested. The method has been checked by tests on one gutter.

3 . Theoretical formulas, derived after certain simplifying assumptions, have been found for level rectangular, semicircular, triangular, and trapezoidal channels where the quantity flowing is proportional to the distance from one end of the channel. The theoretical results are compared briefly with the experimental data in the case of the rectangular channels.

Washington, October 20, 1933. 